\title{
Oxaliplatin-Induced Peripheral Neuropathy Grade in Females with Colorectal Cancer Patients
}

\author{
Inas M. M. Ahmed ${ }^{\mathrm{a}}$, Lamia M. El Wakeel ${ }^{\mathrm{a} *}$, Amr S. Tawfik $^{\mathrm{b}}$, Raafat R. Abdel-Malek ${ }^{\mathrm{c}}$ \\ ${ }^{a}$ Department of Clinical Pharmacy, Faculty of Pharmacy Ain Shams University, Cairo, Egypt \\ ${ }^{b}$ Department of Oncology, Faculty of Medicine, Ain Shams University, Cairo, Egypt \\ ${ }^{c}$ Department of Oncology, Faculty of Medicine, Cairo University, Cairo, Egypt
}

\begin{abstract}
The oxaliplatin-based regimen is considered an important standard of care for patients with colon cancer (CC). Neurotoxicity is the most relevant non-hematologic toxicity of oxaliplatin. The current study aimed to assess the association between colorectal cancer patient's (CRC) gender and susceptibility of oxaliplatin-induced sensory and motor peripheral neuropathy grades in Egyptian patients. All eligible patients between 18-75 years old with CRC were included in the study and only those who had a neurologic disease or diseases impairing neurologic function were excluded. Then eligible patients were stratified into two groups according to gender; oxaliplatin-induced peripheral neuropathy (OIPN) was evaluated and graded on day 1 of each chemotherapy cycle for 12 cycles according to the National Cancer Institute Common Terminology Criteria for Adverse Events (NCI-CTCAE), version 4.0 from March 2015 to October 2017. From a total of 47 females and 33 males; 41(87.2\%) females and 29 (87.9\%) males suffered from different grades of sensory and motor peripheral neuropathy. Regarding sensory oxaliplatin-induced peripheral neuropathy (OIPN), females had a higher risk of grade II-III PN than males (92.7\% versus $58.6 \%$ respectively; $p=0.001)$ For motor OIPN, females were more susceptible to grade IIIII PN versus males ( $84.6 \%$ versus $56.5 \%$ respectively; $\mathrm{p}=0.015)$. In conclusion, High grade (grade II-III) Oxaliplatin-induced sensory and motor peripheral neuropathy occurred more frequently in females versus males Egyptian colorectal cancer patients.
\end{abstract}

Keywords: Colorectal cancer; oxaliplatin-induced peripheral neuropathy; females; males.

*Correspondence | Lamia M. El Wakeel; Department of Clinical Pharmacy; Faculty of Pharmacy Ain Shams University, Cairo, Egypt.

Email: lamywak@yahoo.com

Citation | Ahmed IM, El Wakeel LM, Tawfik A S, Abdel-Malek R R. 2019. Oxaliplatin-Induced High Peripheral Neuropathy Grades in Females with Colorectal Cancer Patients. Arch Pharm Sci ASU 3(1): 116-122

DOI: 10.21608 /aps.2019.10411.2020

Online ISSN: 2356-8380. Print ISSN: 2356-8399.

Received 01 May 2019. Accepted 23 May 2019.

Copyright: ${ }^{\circledR} 2019$ Ahmed et al. This is an open-access article licensed under a Creative Commons Attribution 4.0 International License (CC BY 4.0), which permits unrestricted use, distribution, and reproduction in any medium, provided the original author(s) and source are credited Published by: Ain Shams University, Faculty of Pharmacy

\section{INTRODUCTION}

Oxaliplatin is the third-generation platinum drug which acts by forming DNA adducts and crosslinks that result in inhibition of the cell replication and promoting apoptosis. Moreover, Oxaliplatin is considered as a standard treatment for locally advanced and metastatic colorectal cancer in conjunction with 5-fluorouracil/ 
leucovorin (5-FU/LV). The drug has shown an improvement in survival rate in the adjuvant setting among Stage III patients in comparison to 5-FU/ LV treatment as well as in the first-line treatment of metastatic cancers, compared with 5FU/irinotecan therapy [1-2]. Peripheral neuropathy is one of the most common complications of oxaliplatin chemotherapy [3].Over $70 \%$ of the patients treating by oxaliplatin suffering from some degree of sensory neuropathy according to food and drug administration (FDA)[4].The incidence of peripheral neuropathy induced by oxaliplatin correlates greatly with certain factors include; dose and duration of oxaliplatin [5-6], genetic factors [7], patients preexisting neuropathy and age-related axonal loss[8-9].

In international literature, there is no data on chemotherapy peripheral neuropathy concerning gender available till now, so the current study trying to address these issues.

The current study was designed to assess the association between the gender of colorectal cancer patient's (CRC) and the occurrence of oxaliplatin-induced sensory and motor peripheral neuropathy with its various grades in Egyptian patients.

\section{PATIENTS AND METHODS}

A prospective, observational cohort study was conducted on colorectal cancer patients who were scheduled to receive oxaliplatin dose chemotherapeutic regimen.

We collected the data for eighty colorectal cancer patients between 18-80 years who received oxaliplatin in the different regimen (FOLOFOX4 and FOLOFOX6) at the Oncology and Nuclear Medicine Department at Ain Shams University Hospitals. The patients were assessed for eligibility. After meeting the eligibility criteria, Eligible patients were fully informed of the study design and protocol. Only eighty patients, who accepted to participate and signed informed consent, were included in the study. All consented patients were assigned in the study received oxaliplatin regimen as part of their chemotherapy protocol for colorectal cancer, then the patients were followed up for 6 months and evaluated the incidence and severity of peripheral neuropathy induced by oxaliplatin chemotherapy in males versus females.

The current study excluded only patients with neurologic disease or a disease impairing neurologic function at baseline.

The study protocol was revised and approved by the Committee of Ethics, Faculty of Pharmacy, Ain Shams University (serial number:38).

\subsection{Clinical evaluation of neurotoxicity}

All colorectal cancer patients free from signs or symptoms of peripheral neuropathy treated by oxaliplatin containing regimen (FOLFOX4 and FLOFOX6) were evaluated at baseline before inclusion in the study. The patients follow up was done using follow-up care for patients' self-assessment and daily reporting of the occurrence of sensory/motor peripheral neuropathy. The patients were assessed for sensory and motor OIPN every 2 weeks (every cycle) for 12 cycles (6 months period). At baseline; patients were educated about how to use the follow-up card and how to report the expected signs and symptoms. The symptoms reported were tingling, numbness, weakness, burning sensation, stabbing like pain, loss of sensation and hypersensitivity to mild painful stimuli, cold-induced painful dysesthesia, motor weakness, and pain characteristics. The incidence and severity of peripheral neuropathy sensory and motor-related to oxaliplatin were assessed after each cycle following the National Cancer Institute Common Terminology Criteria for Adverse 
Events (NCI-CTCAE), version 4.0. At the end of the study, oxaliplatin-induced grade II-III sensory and motor neuropathy (high grade) were compared to grade I (low grade) sensory and motor neuropathy in males versus females.

The highest grade of sensory/motor peripheral neuropathy attained by patients at any particular cycle was recorded as the OIPN for that cycle.

\subsection{NCI-CTC for Neuropathy}

* Peripheral Sensory Neuropathy

* Grade I: Asymptomatic without interfering with the function

* Grade II: Sensory alteration interfering with function without ADL

* Grade III: Sensory alteration interfering with ADL

* Grade IV: Life-threatening consequence (Disabling)

* Grade V: Death

* Peripheral Motor Neuropathy

* Grade I: Asymptomatic, weakness by exam/testing only

* Grade II: Symptomatic weakness interfering with function without interfering with ADL

* Grade III: Weakness interfering with ADL

* Grade IV: Life-threatening

* Grade V: Death

* Abbreviations: ADL, activities of daily living

\subsection{Statistical analysis}

Numerical data were expressed as mean and standard deviation or median and range. While qualitative data were expressed as frequency and percentage. Chi-square test or Fisher's exact test was used to examine the relation between qualitative variables. Wilcoxon-signed ranks test (non-parametric paired t-test) was used to compare two consecutive measures of not normally distributed numerical variables. All tests were two-tailed. A p-value $<0.05$ was considered significant. The Statistics were performed using IBM $\odot$ SPSS $\odot$ Statistics version 22 (IBM@ Corp., Armonk, NY, USA)

\section{RESULTS}

From March 2015 till October 2017, all colorectal cancer patients attending the clinic of Oncology and Nuclear Medicine Department, Ain Shams University Hospitals, Cairo, Egypt. Were evaluated and those who fit the inclusion criteria were included in the study.

Out of 120 patients screened, only 80 patients, fulfilled the inclusion criteria and were included in the study. The patients were stratified according to gender to Female group and male group. The main clinical data of colorectal cancer patients according to gender represented in Table 1.

\subsection{OIPN in females versus males}

From a total of 47 females and 33 males; 41(87.2\%) females and 29 (87.9\%) males suffered from different grades of sensory and motor peripheral neuropathy.

For sensory OIPN, the females were associated with a higher risk of grade II-III OIPN than males ( $92.7 \%$ versus $58.6 \%$ respectively; $\mathrm{p}=0.001)$. For motor OIPN, the females were more susceptible to grade II-III than males $(84.6 \%$ versus $56.5 \%$ respectively; $\mathrm{p}=0.015$ ).

The percentage of Sensory and motor peripheral neuropathy different grades in females versus males are represented in Table 2 and Figs. 1 and 2. 
Table 1: Clinical data of colorectal cancer patients in females versus males

\begin{tabular}{|c|c|c|c|}
\hline & Females & Males & $\overline{p \text {-value }}$ \\
\hline \multicolumn{4}{|l|}{ Age (years) } \\
\hline \multirow[t]{2}{*}{ mean \pm SD } & $46.8 \pm 10.7$ & $46.9 \pm 11.2$ & \multirow{3}{*}{0.754} \\
\hline & & & \\
\hline (Range) & $(26-73)$ & $(25-65)$ & \\
\hline \multicolumn{4}{|c|}{ Dose $\left(\mathrm{mg} / \mathrm{m}^{2}\right) /$ Cycle } \\
\hline mean \pm SD & $139 \pm 18.7$ & $148.5 \pm 23.2$ & \multirow{3}{*}{0.072} \\
\hline \multirow[t]{2}{*}{ (Range) } & & & \\
\hline & $(90-170)$ & $(100-230)$ & \\
\hline \multicolumn{4}{|c|}{ Weight (Kg) } \\
\hline \multirow[t]{2}{*}{ mean $\pm \mathrm{SD}$} & $64.8 \pm 14.8$ & $69.9 \pm 14.5$ & \multirow{3}{*}{0.214} \\
\hline & & & \\
\hline (Range) & $(38-114)$ & $(50-104)$ & \\
\hline Total & 47 & 33 & - \\
\hline
\end{tabular}

* P-values less than 0.05 are considered significant

Note: $\mathrm{Kg}$, kilogram; $\mathrm{mg} / \mathrm{m}^{2}$, milligram per meter $^{2}$; $\mathrm{SD}$, standard deviation

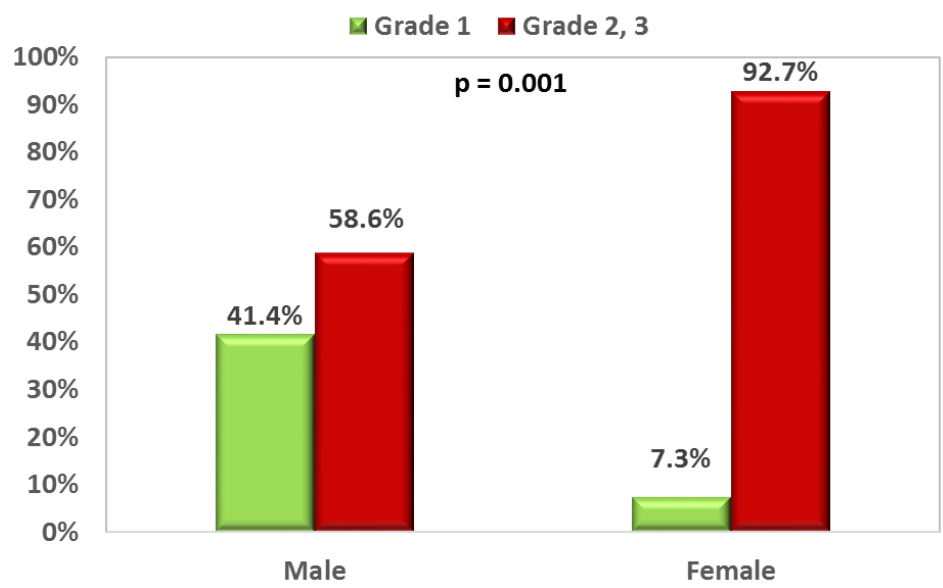

Fig. 1. Association of sensory peripheral neuropathy induced by oxaliplatin chemotherapy in male versus female with colorectal cancer. The statistical significance of the results was analyzed using the Pearson Chi-Square test $(\mathrm{p}=0.001)$. 
Table 2. Sensory and motor peripheral neuropathy different grades in females versus males with colorectal cancer patients

\begin{tabular}{lllll}
\hline Parameter & Category & \multicolumn{2}{c}{ Gender } & P-value \\
& & Males & Females & \\
\hline Sensory & G I & 12 & $3(7.3 \%)$ & 0.001 \\
Neuropathy; & & $(41.4 \%)$ & & \\
G 0 $=10(12.5 \%)$ & G II-III & $17(58.6 \%)$ & $38(92.7 \%)$ & \\
& Total & 29 & 41 & \\
Motor neuropathy; & N;70(87.5\%) & & & \\
G0 $=18(22.5 \%)$ & G I & $10(43.5 \%)$ & $6(15.4 \%)$ & 0.015 \\
& G II-III & $13(56.5 \%)$ & $33(84.6 \%)$ & \\
& Total & 23 & 39 & \\
& N:62(77.5\%) & & & \\
& & & & \\
& & & & \\
& & & & \\
& & & & \\
& & & & \\
\end{tabular}

*P-values 0.001 is considered significant

Note: G, Grade of peripheral neuropathy; n, number

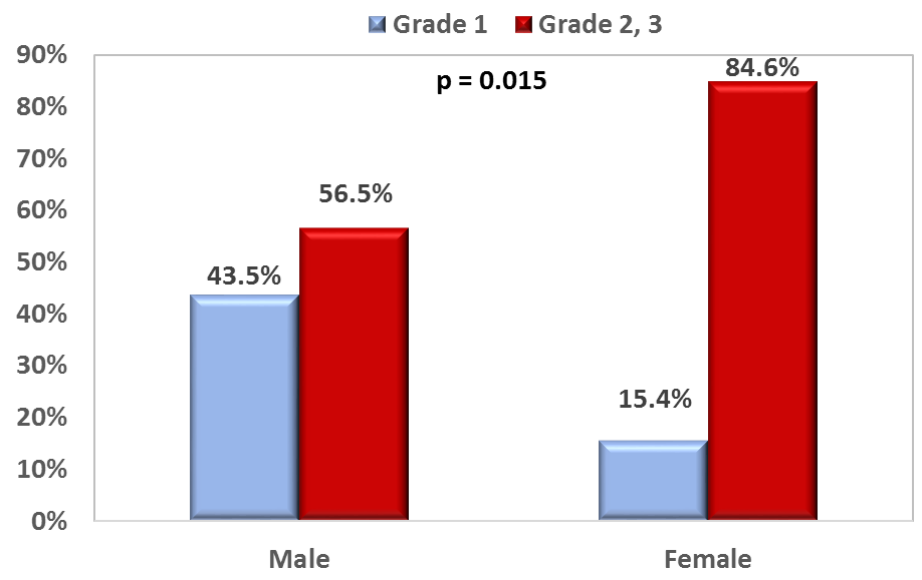

Fig. 2. Association of motor peripheral neuropathy induced by oxaliplatin chemotherapy in male versus female with colorectal cancer. The statistical significance of the results was analyzed using the Pearson Chi-Square test ( $\mathrm{p}=0.015)$.

\section{DISCUSSION}

Results of this prospective study of eighty colorectal cancer patients have shown that female patients were more significantly affected by peripheral neuropathy (grade II-III) sensory and motor than males. Sensory peripheral neuropathy was significantly higher $(92.7 \%)$ in females compared with males (58.6\%). While in motor peripheral neuropathy, the females were also more affected $(84.6 \%)$ than males $(56.5 \%)$. To the best of our knowledge, this is the first study to 
date carried out data on oxaliplatin peripheral neuropathy reference to gender.

Previous studies in chemotherapy-induced peripheral neuropathy had not compared the risk of PN among gender. One previous cohort study correlated the peripheral neuropathy with the gender of patients with human immunodeficiency virus (HIV) who were treated with antiretroviral therapy (ART) and found that the incidence was ten times higher in females than males to develop PN in the first year of ART [4]. There are no absolute reasons clearly explained for such greater adverse events in females. However, suggested mechanisms for gender differences have included differences in fat compositions or body mass index and changing in hormonal states that may affect the distribution and the metabolism of the drug [10].

On the contrary to the current results, Fraser et al. showed that there was no consistent relationship exists between the onset of neuropathy and age, sex and duration of diabetes [11]. Another study also showed that genderbased differences in diabetic peripheral neuropathy are statistically not significant with age at diagnosis of diabetes or the onset of diabetic peripheral neuropathy [12].

Previous studies have shown that the neurotoxic side effects of oxaliplatin included acute, transient neuropathy which occurs in about $90 \%$ of the patients within hours of infusion and is suffering from paresthesias of the hands, feet and dysesthesias These symptoms are usually induced by exposure to lower degrees of temperatures and significant features of oxaliplatin-induced cold allodynia[13]. Moreover, motor symptoms involving prolonged muscular contractions commonly happen [14]. Between the treatment cycles of the oxaliplatin, the acute neuropathy mainly subsides [15]. the continuing exposure to oxaliplatin can develop severe chronic neuropathy in approximately $70 \%$ of patients if the accumulative doses exceeding $540 \mathrm{mg} / \mathrm{m}^{2}$ [13][16]. Clinically, the symptoms of chronic oxaliplatin-induced neuropathy closely are similar to those of the acute condition and include temperature-insensitive paresthesias and dysesthesias of the limbs. furthermore, changes in proprioception, which may affect normal daily activities requiring fine motor coordination, reportedly occur at accumulative doses exceeding $780 \mathrm{mg} / \mathrm{m}^{2}$ [14][16].

\section{Limitations of the study}

1. A small sample size of patients.

2. The study conducted at a single center organization.

\section{Conclusion}

Oxaliplatin induced high-grade II-III sensory and motor peripheral neuropathy seems to occur more frequently in female's gender than males in Egyptian colorectal cancer patients. Findings of the current study warrant strict adverse effect monitoring of females receiving Oxaliplatin to guard against the occurrence of neuropathy.

\section{Recommendations}

1. The present study provides evidence in OIPN in female's patients more than in males. The further examination correlated between gender and the occurrence of peripheral neuropathy it's recommended in a larger sample.

2. Use objective evaluation methods to evaluate peripheral neuropathy.

3. The study should conduct at the multicenter organization.

\section{Conflict of interest}

The authors declare that they have no conflicts of interest.

Funding Statement: not applicable 


\section{REFERENCES}

1. Goldberg RM, Sargent DJ, Morton RF, Fuchs CS, Ramanathan RK, Williamson SK, and Alberts SR. A randomized controlled trial of fluorouracil plus leucovorin, irinotecan and oxaliplatin combinations in patients with previously untreated metastatic colorectal cancer. J Clin Oncol 2004; 22(1): 23-30.

2. Berretta M, Lleshi A, Di-Benedetto F, Bearz A, Spina M, Tirelli U. Oxaliplatin and capecitabine (Xelox) in association with highly active antiretroviral therapy in advanced hepatocarcinoma HIV/HCV-infected patients. Ann Oncol 2006; 17(7): 1176-1177.

3. Griffith KA, Zhu S, Johantgen M, Kessler MD, Renn C, Beutler AS, Kanwar R, Ambulos N, Cavaletti G, Bruna J, Briani C, Argyriou AA, Kalofonos HP, Yerges-Armstrong LM, Dorsey SG. Oxaliplatin-Induced Peripheral Neuropathy and Identification of Unique Severity Groups in Colorectal Cancer. J Pain Symptom Manage 2017; 54(5):701-706.

4. Mehta SA, Ahmed A, Laverty M, Holzman RS, Valentine $F$, and Sivapalasingam S. Sex differences in the incidence of peripheral neuropathy among Kenyans initiating antiretroviral therapy. Clin Infect Dis 2011; 53(5): 490-496.

5. Extra, J. M., M. Espie, F. Calvo, C. Ferme, L. Mignot, and M. Marty. Phase I study of oxaliplatin in patients with advanced cancer. Cancer Chemother Pharmacol 1990: 125(4): 299303.

6. Fardell JE, Vardy J, Monds LA, Johnston IN. The long-term impact of oxaliplatin chemotherapy on rodent cognition and peripheral neuropathy. Behav Brain Res 2015; 291:80-88.

7. Palugulla S, Devaraju P, Kayal S, Narayan SK, Mathaiyan J. Genetic polymorphisms in cyclin $\mathrm{H}$ gene are associated with oxaliplatin-induced acute peripheral neuropathy in South Indian digestive tract cancer patients. Cancer Chemother Pharmacol. 2018;82(3):421-428.

8. Chaudhry V, Chaudhry M, Crawford TO, SimmonsO'Brien E, and Griffin JW. Toxic neuropathy in patients with pre-existing neuropathy. Neurology 2003:60(2): 337-340.

9. Tanishima H, Tominaga T, Kimura M, Maeda T, Shirai Y, Horiuchi T. Hyperacute peripheral neuropathy is a predictor of oxaliplatin-induced persistent peripheral neuropathy. Support Care Cancer 2017;25(5):1383-1389

10. Ofotokun I and Pomeroy C. Sex differences in adverse reactions to antiretroviral drugs. Top HIV Med 2003;11(2):55-9.

11. Fraser DM, Campbell IW, Ewing DJ, and Clarke BF. Mononeuropathy in diabetes mellitus. Diabetes 1979. 28(2): 96-101.

12. Javed A, Furqan A, Zaheer M and Kasuri N. Gender-based differences in diabetic peripheral neuropathy. PJNS 2014; 9(4):20-24.

13. Argyriou AA, Briani C, Cavaletti G, Bruna J, Alberti P, Velasco R and Santos C. Advanced age and liability to oxaliplatin-induced peripheral neuropathy: post hoc analysis of a prospective study. Eur J Neurol 2013; 20(5): 788-794.

14. Boyette-Davis JA, Hou S, Abdi S, Dougherty PM. An updated understanding of the mechanisms involved in chemotherapy-induced neuropathy. Pain Manag 2018; 8(5): 363-375

15. Extra JM, Marty M, Brienza S and Misset JL. Pharmacokinetics and safety profile of oxaliplatin. Semin Oncol; 1998, 25(2): 13-22.

16. Cersosimo RJ. Oxaliplatin-associated neuropathy: a review. Ann Oncol 2005; 39(1):128-135. 